\title{
Geometric regeneration and mechanical analysis of a gas turbine blade type Frame 9001 GE
}

\author{
Salah Amroune ${ }^{a^{*}}$, Barhm Mohamad ${ }^{\mathrm{b}}$, Mustafa Moussaouic ${ }^{\mathrm{c}}$ and Hafida Saaidi ${ }^{\mathrm{a}}$
}

${ }^{a}$ Université Mohamed Boudiaf; B.P 166 ICHBELIA M'sila 28000, Algérie

${ }^{b}$ Petroleum Technology Department, Koya Technical Institute, EPU, KRG, Iraq

cUniversité Ziane Achour; B.P 3117 Djelfa 17000, Algérie

\begin{tabular}{l}
\hline A R T I C L EI N F O \\
\hline Article history: \\
Received 22 September, 2017 \\
Accepted 10 January 2018 \\
Available online \\
18 March 2018 \\
\hline Keywords: \\
Geometric regeneration \\
Gas turbine blade \\
Structural analyses \\
Energy elastic
\end{tabular}

\section{A B S T R A C T}

\begin{abstract}
Simulation and visualization of the mechanical components have become a predominant phase during the design and the production stages. Several means are used to improve the design and to reduce study time. Today, the powerful hardware and the software available on the market have contributed greatly on the improvement of design, visualization and manufacturing process of complex parts (turbine blade). In this context, our study is a contribution to the establishment of a methodology to a CAD modelling and finite element analysis, which allows us to identify the mechanical behavior of a gas turbine blade. The profile of the blade turbine model is obtained after regeneration using the CATIA V5R20 software from the retro-design technique using a FARO-type scanner. The turbine blade is analyzed under a static mechanical behavior. It has been observed that the maximum stresses and deformations are located in the vicinity of the root and the upper surface along the turbine blade. On the other hand, the elastic energy is located at a distance from the root of the turbine blade.
\end{abstract}

(C) 2018 Growing Science Ltd. All rights reserved.

\section{Introduction}

The turbine blades are the individual components that make up the turbine of a gas turbine engine and convert the kinetic energy of the burning gases into mechanical energy. It is the vanes that condition the efficiency of the turbine. In fact, the modelling of the damages and the forecasting of their lifetimes constitute a main research axis. The turbine blades of the warmer stages are those parts which are subjected to the most damaging stresses due to numerous factors such as the very high temperature of the burnt gases (often higher than the melting temperature of the alloys), the strong thermal gradients present during the take-off and landing phases in the case of aircraft engines, creep of centrifugal force, hot corrosion, high stresses induced by complex geometry and vibration fatigue. But these conditions also varies over time during the mission of the engine, therefore, require a high level of mechanical properties (fatigue-creep) and an environmental stability (resistance to oxidation, corrosion) (Loranchet, 1992).

* Corresponding author

E-mail addresses: amroune salah@yahoo.fr (S. Amroune) 
The complexity of these parts requires a perfect integration between design, materials and manufacturing techniques. The vanes are therefore made of a chromium-modified aluminium-coated nickel-based super alloy. At the leading edge, the severity of the local thermal loading can constitute privileged sites of damage (Xie et al., 2006; Boyaraju et al., 2015; Padture et al., 2002). Work has been carried out on turbine blade failed using metallurgical examinations which include activities such as determination of the composition of the material, visual inspection and microscopic examination (Mazur et al., 2015; Hou et al., 2002; Vardar \& Ekerim, 2007; Kermanpur et al., 2008; Carter, 2015).

Rao et al. (2014) presented a research that summarizes an attempt that was made to analyse the failure of the gas turbine blade made of nickel base superalloys by mechanical analysis. The mechanical analysis was carried out assuming that there could be a failure in the material of the blade due to the high temperatures and the large supported centrifugal forces which can ultimately lead to a final rupture of the gas turbine blade. A recent study by Mohamad and Abdelhussien (2016) allows the analysis of failure of the turbine blade of a type of gas turbine 9E GE installed in a certain type of simple systems constituted by the gas turbine driving an electric power generator. A calculation was also carried out with an excessive speed. The focus of this study is on the damage mechanisms of the turbine blade and critical areas of high stress.

Our research focuses mainly on structural static analysis and the design of a 3D model of the turbine blade based on data from a FARO type laser scanner. The technique of retro design is adopted to generate 3D surface data of the turbine blade. The profile of the gas turbine blade model obtained is generated using the CATIA V5R20 software. The 3D model of a gas turbine blade with root was carried out in two stages. These were then combined to create a single volume. The turbine blade is analysed for its mechanical performance. A static analysis was performed to find out the mechanical stresses and deformations of the gas turbine rotor blades, which include parameters such as gas forces that are assumed to be evenly distributed, tangential and axial forces acting through the centroid of the blade. The centrifugal force also acts through the centroid of the blade in the radial direction.

\section{Material and method}

\subsection{Properties of a gas turbine blade studied}

The blade studied belonging to $110 \mathrm{MW}$ gas turbine engines used for the production of electrical energy with a turbine gas inlet temperature of about $1350^{\circ} \mathrm{C}$. The turbine blades were super nickelbased alloys having excellent mechanical strength and good creep resistance at high temperature (typically 0.7 to 0.8 times its melting temperature), good surface stability and good resistance to corrosion and oxidation, they are manufactured according to the high pressure molding method. The chemical composition of the turbine blade is determined by means of an apparatus Thermo Fisher Scientific and is presented in Table 1, the chemical analysis of the blade allows to identify the material which corresponds to the grade $46 \mathrm{Ni}-8 \mathrm{Cr}-44 \mathrm{Fe}-0.5 \mathrm{Co}$. Fig. 1 shows the blades to be studied, the latter belonging to the blades of the gas turbine compressor part of the Frame 9001 GE type.

Table 1. Chemical composition of gas turbine blade

\begin{tabular}{|c|c|c|c|c|c|c|c|c|c|}
\hline Element & $\mathrm{Sn}$ & Mo & $\mathrm{Nb}$ & $\mathrm{Ni}$ & $\mathrm{Co}$ & $\mathrm{Fe}$ & $\mathrm{Mn}$ & $\mathrm{Cr}$ & $\mathrm{V}$ \\
\hline $\mathrm{Wt} \%$ & 0.011 & 0.084 & 0.003 & 46.366 & 0.404 & 44.285 & 0.488 & 8.279 & 0.030 \\
\hline Error & \pm 0.005 & \pm 0.003 & \pm 0.001 & \pm 0.114 & \pm 0.052 & \pm 0.096 & \pm 0.034 & \pm 0.047 & \pm 0.006 \\
\hline
\end{tabular}

\subsection{Modelling of the gas turbine blade}

The blade examined belonging of the blade of the $110 \mathrm{MW}$ gas turbine compressor rotor to be operated in a power plant (SPE M'sila, Algeria) to reproduce electrical energy. The technique of retro design is applied to generate 3D surface data of the turbine blade. This technique uses a FARO type laser scanner (Fig. 2) to acquire the data in the form of point clouds (1380675 points) in a text file which groups the acquired data in the form of three coordinates $\mathrm{x}, \mathrm{y}$ and $\mathrm{z}$. 


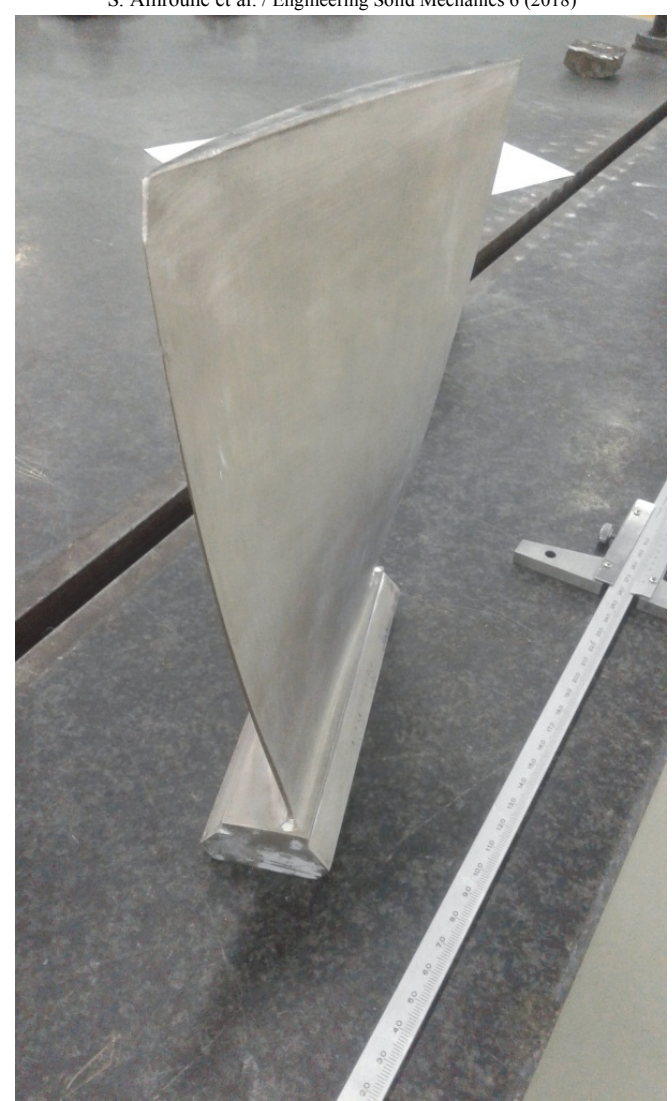

Fig. 1. Gas turbine blade Frame 9001 GE.

The profile of the gas turbine blade model is generated using the CATIA V5R20 software. The 3D model of a gas turbine blade with root was made in two steps (Digitized Shape Editor, Quick Surface Reconstruction). These two were then combined to create a single volume. The geometric model of the gas turbine blade is illustrated in Fig. 3.
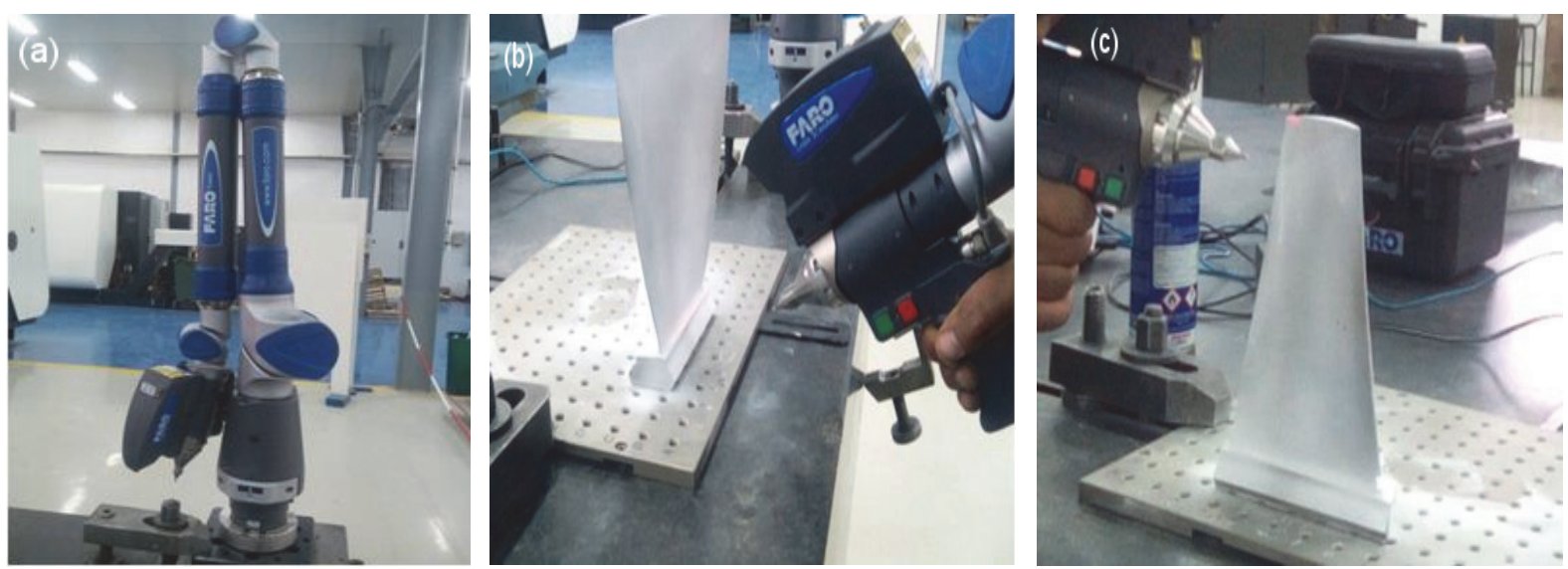

Fig. 2. Acquisition of data: (a) FARO type scanner used for data acquisition (MEI SONELGAZ M'sila, Algérie), (b) start of scan and (c) end of scan 


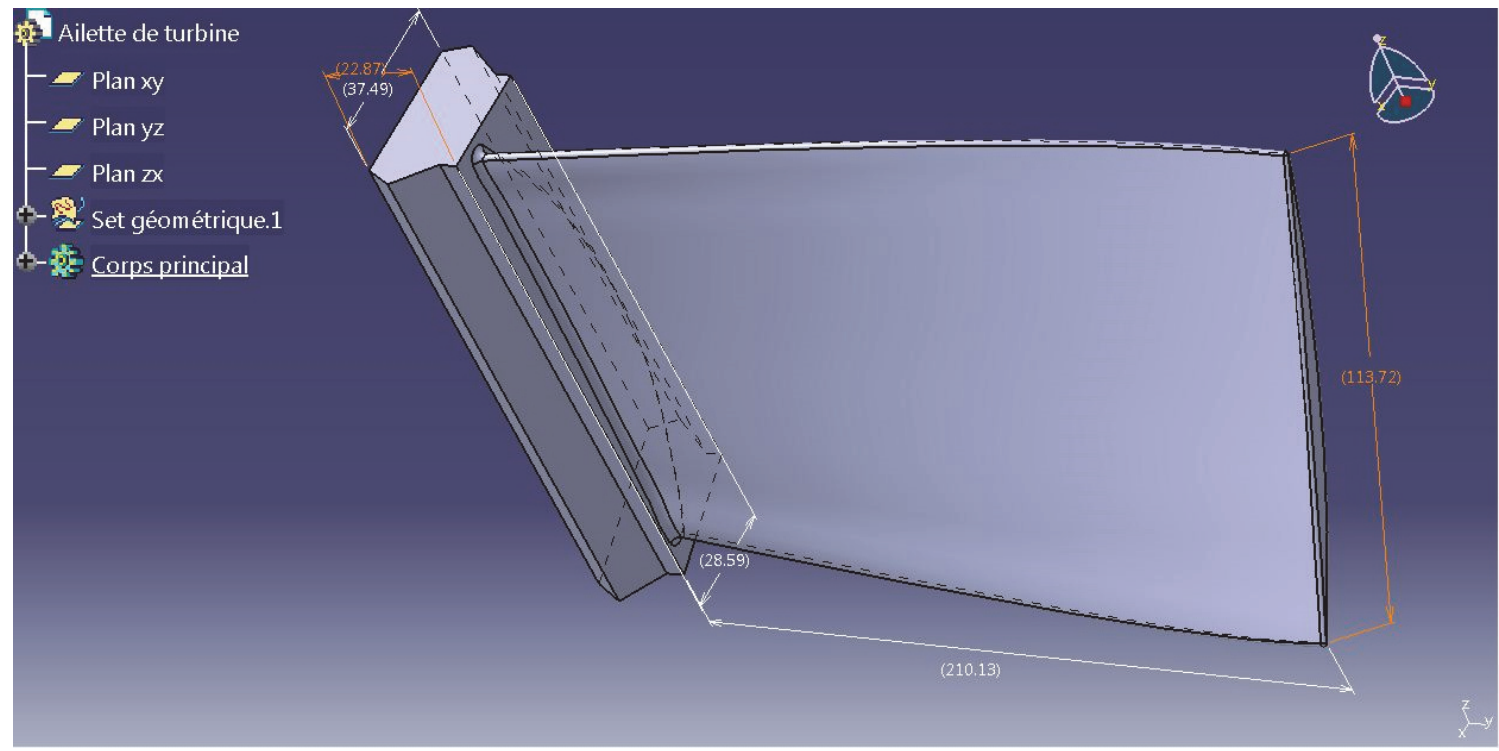

Fig. 3. Geometric model of gas turbine blade using CATIA V5R20

\subsection{Finite Element Analysis of a Gas Turbine Blade}

The analysis of the stress distribution in the field of gas turbine engineering is invariably complex and for many problems it is extremely difficult and tedious to obtain analytical solutions. The finite element method is a numerical analysis technique to obtain approximate solutions. It has now become a very important and powerful tool for the digital solution of a wide range of engineering problems. The method used for the analysis of solid structures of complex shapes and complicated boundary conditions.

The turbine blade is analysed under static mechanical behavior using the CATIA V5R20 software. The compressor rotor of the gas turbine Frame 9001 GE has 32 fins distributed around the circumference of the rotor wheel. Only one turbine blade is taken into consideration for the analysis. The cross-section of the blade is in the $\mathrm{X}-\mathrm{Z}$ plane and the length of the blade is along the Y-axis.

A static analysis was performed to determine the mechanical stresses and deformations undergone by the rotor blade of the gas turbine. In this analysis, it is assumed that the gas forces are uniformly distributed their resultant is decomposed into tangential $(\mathrm{Ft})$ and axial forces $(\mathrm{Fa})$ acting through the centroid of the blade.

The rotor blades suffer the effects of centrifugal force, aerodynamics, chemical and thermal actions of gases and vibrations. The centrifugal force also acts through the center of the blade in the radial direction. It is given by the Eq. (1):

$$
F_{\mathrm{c}}=m \cdot w^{2} \cdot R=\frac{V^{2}}{R}
$$

where :

$\mathrm{m}$ : mass of the turbine blade;

$\mathrm{W}$ : Rotation speed of the rotor;

$\mathrm{R}$ : distance between the centroid of the turbine blade and the axis of rotation.

Centrifugal force $(\mathrm{Fc})=281661.5 \mathrm{~N}$

The components of the resultant of the gas forces are evaluated by constructing velocity triangles at the inlet and outlet of the rotor blades. This decomposition resulted in two forces, one tangential and one axial:

Tangential forces $(\mathrm{Ft})=780 \mathrm{~N}$ 
The type of mesh used for the gas turbine blade during the analysis process in CATIA V5R20 is a parabolic mesh (Fig. 4). The mesh characteristics are given in Table 2.

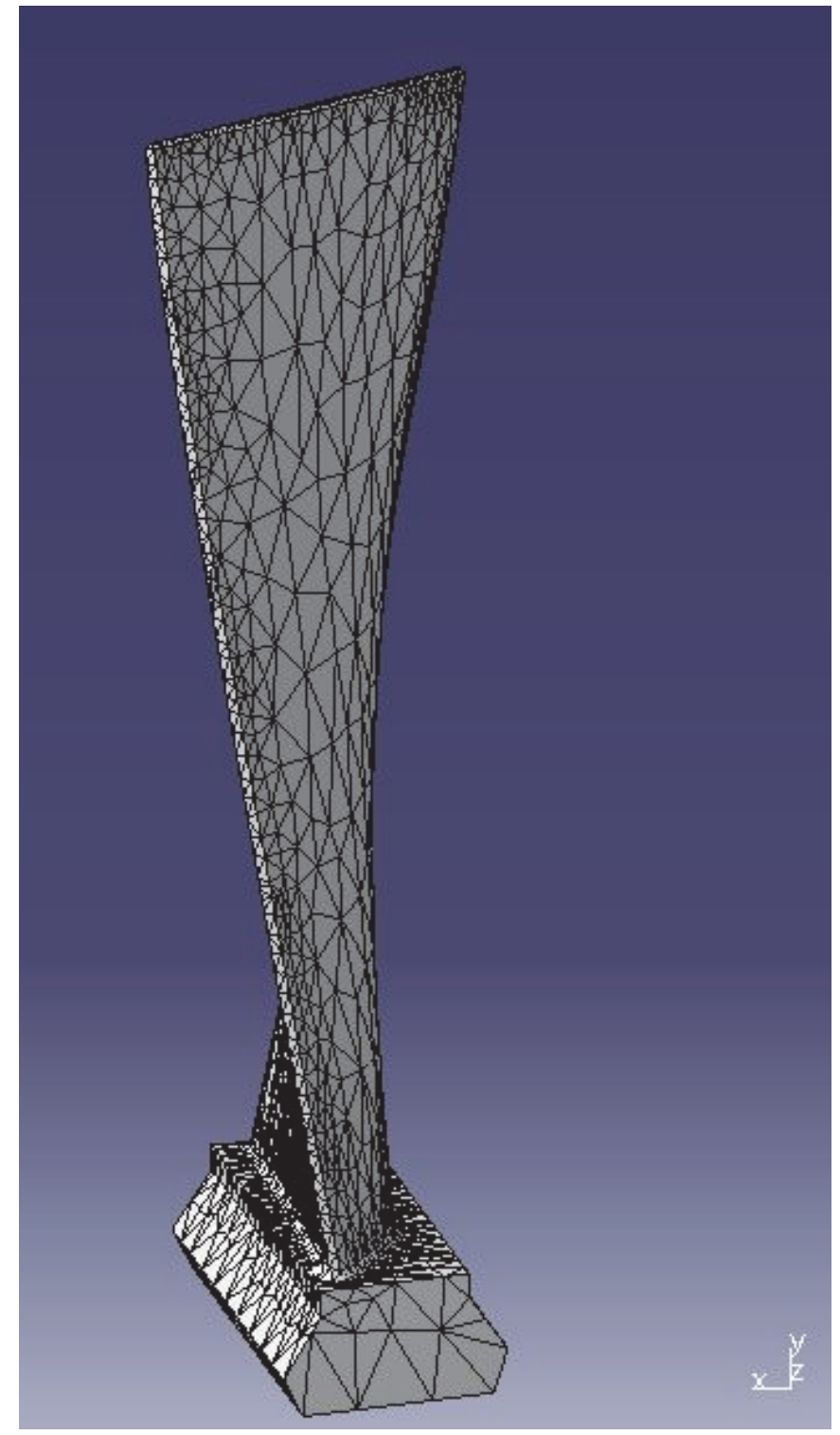

Fig. 4. Meshing of turbine blade under analysis

Table 2. The mesh characteristics

\begin{tabular}{lllllll}
\hline $\begin{array}{l}\text { Type } \\
\text { of mesh }\end{array}$ & Element type & $\begin{array}{l}\text { Intermediate node } \\
\text { parameters }\end{array}$ & $\begin{array}{l}\text { Element Size } \\
(\mathrm{mm})\end{array}$ & $\begin{array}{l}\text { Total number of } \\
\text { nodes }\end{array}$ & $\begin{array}{l}\text { Total number of } \\
\text { items }\end{array}$ & $\begin{array}{l}\text { Geometric } \\
\text { tolerance } \\
(\mathrm{mm})\end{array}$ \\
\hline $\begin{array}{l}\text { Octree } \\
\text { 3D }\end{array}$ & Parabolique & $\begin{array}{l}\text { Jacobien=0.3 } \\
\text { Angle=60 }\end{array}$ & 14.518 & 12363 & 6721 & 0.1 \\
\hline
\end{tabular}

\section{Result and discussion}

The rotational movement is communicated to the turbine rotor via the turbine blades which are distributed over the circumference of the wheels. The reception of the forces by the blade coming from the burned gases and the centrifugal forces puts it under bending stress. This situation may involve the 
embedding of one end of the turbine (foot of turbine blade) on one side and on the other side the second end is free (top of the jacket). The stresses induced in the materials of the turbine blade must be within the safe limit after geometrical regeneration of the model (turbine blade) under the software CATIA V5R20, a discretization in domains finite elements are applied. The simulation analysis makes it possible to identify the probable zones of damage through mechanical constraints and deformation parameters. The simulation results obtained revealed significant major deformations of the order (5.52E-6) at the root of the blade (Fig. 5). This zone, where high stress concentration is present, supports an embedding moment and a reaction force. A decrease in the main deformation is indicated far from the foot of the turbine blade.

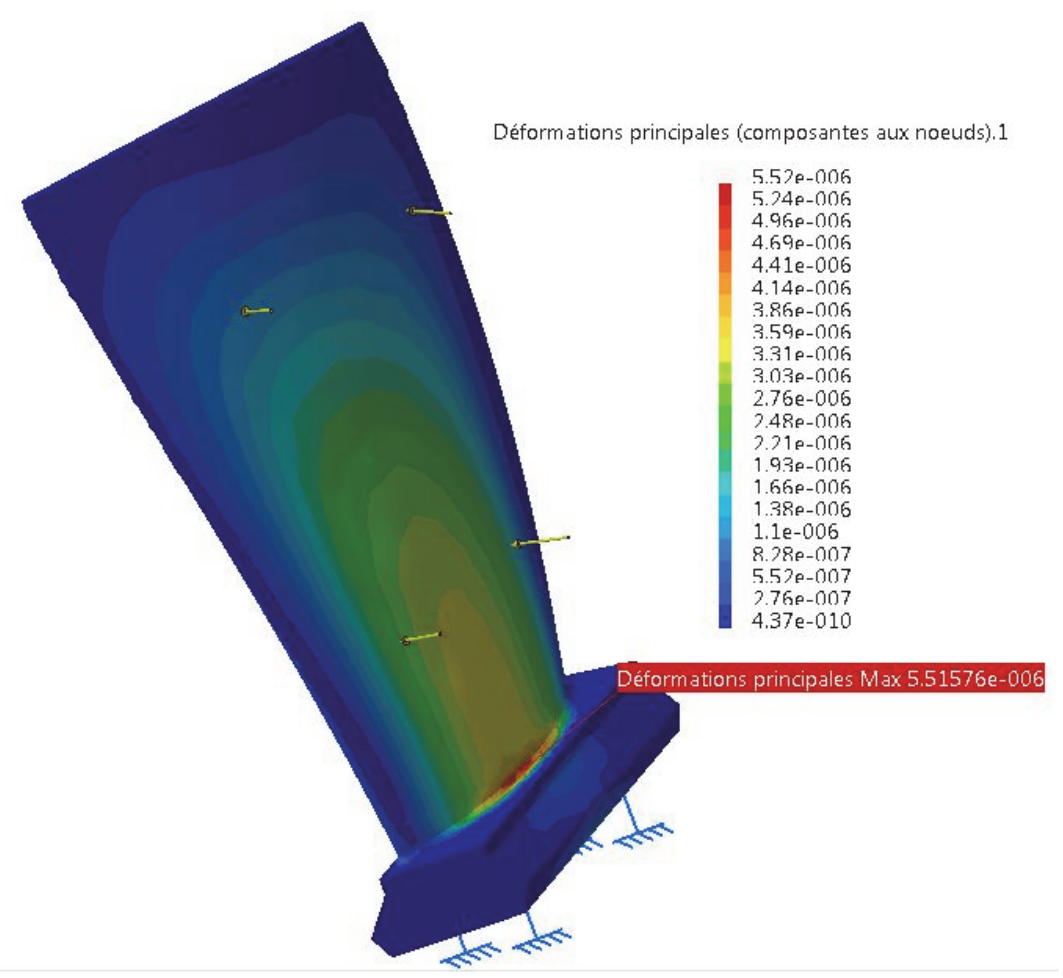

Fig. 5. Main deformation induced stress distribution within gas turbine blade

The combined effect of the main constraints $\sigma_{x}, \sigma_{y}$, et $\sigma_{z}$ is illustrated from the expression of VonMises criterion given by Eq. (2) as follows,

$$
\sigma_{e q}=\frac{1}{\sqrt{2}} \sqrt{\left(\sigma_{x}-\sigma_{y}\right)^{2}+\left(\sigma_{y}-\sigma_{z}\right)^{2}+\left(\sigma_{z}-\sigma_{x}\right)^{2}}
$$

where :

$\sigma_{x}$ : Main constraint by direction $\mathrm{xx}$

$\sigma_{y}$ : Main constraint by direction yy

$\sigma_{z}$ : Main constraint by direction $\mathrm{zz}$

Fig. 6 shows the distribution of Von-Mises stresses along the turbine blade, the maximum (value: $1,015 \mathrm{~N} / \mathrm{mm}^{2}$ ) is located at the root of the blade where the stress gradient is large. Several mechanisms are involved in the failure of the blades under the conditions of operation of the turbine at high speed of rotation at high temperature in a corrosive medium. In operation, the exhaust gases from the combustion chamber are directed through the transition tubes so that the hot gases strike the turbine blades of the first stage HPT, thereby signaling high pressure forces which tend to deform the blade in 
flexion relative to its foot (fir foot). The zone most stressed to the forces induced by the aerodynamic effects is located in the pale-foot blade connection.

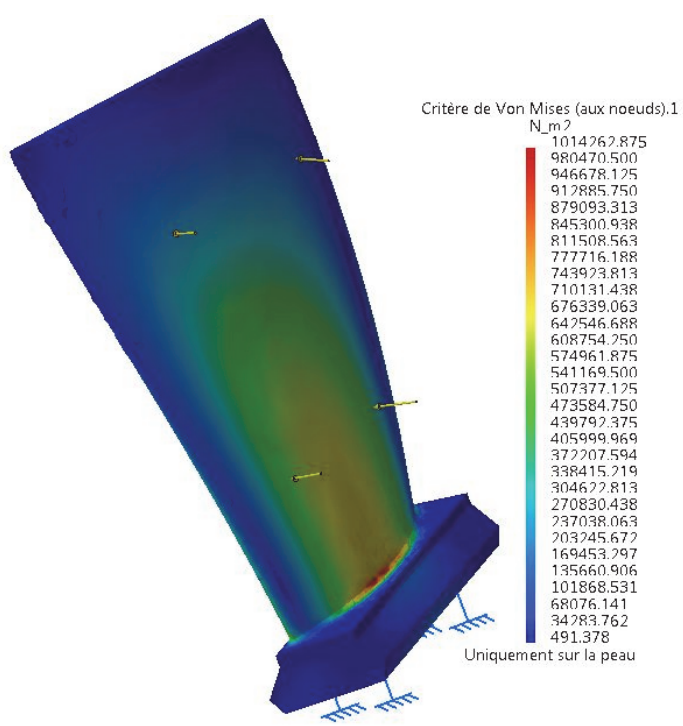

Fig. 6. Von Mises strain analysis

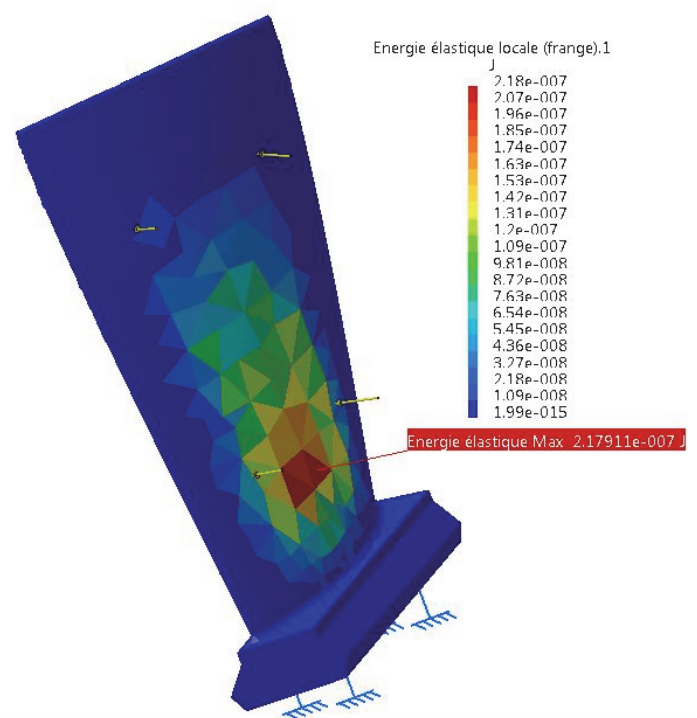

Fig. 7. Local elastic energy contour

Another parameter has been shown to be related to the energy which is the stored local elastic energy given by:

$$
U=\frac{1}{2} \int_{V}\{\epsilon\}^{\mathrm{T}} \cdot\{\sigma\} \cdot d V,
$$

with the components of the stress vector of the solid:

$$
\{\sigma\}^{\mathrm{T}}=\left\{\sigma_{\mathrm{xx}} \sigma_{\mathrm{yy}} \sigma_{\mathrm{zz}} \tau_{\mathrm{xy}} \tau_{\mathrm{yz}} \tau_{\mathrm{xz}}\right\}
$$

and the components of the deformation vector:

$$
\{\varepsilon\}^{\mathrm{T}}=\left\{\varepsilon_{\mathrm{xx}} \varepsilon_{\mathrm{yy}} \varepsilon_{\mathrm{zz}} \gamma_{\mathrm{xy}} \gamma_{\mathrm{yz}} \gamma_{\mathrm{xz}}\right\}
$$

When loading the turbine blade the elastic deformation energy develops from the material near the centre point of the turbine blade which reaches a maximum value of $2.18 \mathrm{E}-7 \mathrm{~J}$ oules (Fig. 7 ). This zone enclosing the centroid point, undergoes a considerable work due to a local deformation of the material.

\section{Conclusion}

In this paper, we studied a geometric regeneration of a gas turbine blade type Frame 9001GE using CATIA software based on finite element analysis. This type of turbine member supports various severe effects that put the turbine blade in a dangerous situation. Based on this study, which enabled us to identify the main points:

- The method of geometric regeneration of the turbine blade using the laser scanner has allowed us to reduce the time enormously and to design the geometry of the model in a precise and complete way and to give a very close physical profile of the turbine blade. This allowed to set up a platform to initiate the various studies (damage, thermal, etc.).

- The number of points obtained during the scanning operation is very important (1380675 points) which requires a very powerful software like CATIA V5R20.

- The analysis carried out on the turbine blade made it possible to identify the probable zones of 
failure where stresses and maximum deformations are located near the root of the blade of the turbine. These parameters (stresses and deformations) are compared with the normalized effective values for the safe holding of the turbine blade. In all other parts of the turbine blade, the induced stresses are within the recommended limits of resistance.

- Local elastic energy is located at a distance away from the root of the blade.

\section{Acknowledgement}

The authors would like to thank Mr. Mustapha Areselan, Engineer at MEI Sonelgaz M'sila (Algeria) for his help in using the FARO type scanner (Fig. 2) and Mr. Vincent, an engineer specializing in CAD (France) for his help.

\section{References}

Boyaraju, G., Rajasekhar, S., Sridhar, A. V., \& Rao, J. H. N. (2015). Thermal analysis of a gas turbine rotor blade. International Journal of Science Engineering and Advance Technology, 3(12), 11811187.

Carter, T. J. (2005). Common failures in gas turbine blades. Engineering Failure Analysis, 12(2), 237247.

Hou, J., Wicks, B. J., \& Antoniou, R. A. (2002). An investigation of fatigue failures of turbine blades in a gas turbine engine by mechanical analysis. Engineering Failure Analysis, 9(2), 201-211.

Kermanpur, A., Amin, H. S., Ziaei-Rad, S., Nourbakhshnia, N., \& Mosaddeghfar, M. (2008). Failure analysis of Ti6Al4V gas turbine compressor blades. Engineering Failure Analysis, 15(8), 10521064.

Loranchet, Y. (1992). Mise en oeuvre des turbines à gaz dans l'industrie. Ed. Techniques Ingénieur.

Mazur, Z., Luna-Ramirez, A., Juárez-Islas, J. A., \& Campos-Amezcua, A. (2005). Failure analysis of a gas turbine blade made of Inconel 738LC alloy. Engineering failure analysis, 12(3), 474-486.

Mohamad, B. A., \& Abdelhussien, A. (2016). Failure Analysis of Gas Turbine Blade Using Finite Element Analysis. International Journal of Mechanical Engineering and Technology, 7(3).

Padture, N. P., Gell, M., \& Jordan, E. H. (2002). Thermal barrier coatings for gas-turbine engine applications. Science, 296(5566), 280-284.

Rao, V. N., Kumar, I. N., Madhulata, N., \& Abhijeet, A. (2014). Mechanical analysis of 1st stage marine gas turbine blade. International Journal of Advanced Science and Technology, 68, 57-64.

Vardar, N., \& Ekerim, A. (2007). Failure analysis of gas turbine blades in a thermal power plant. Engineering Failure Analysis, 14(4), 743-749.

Xie, Y. J., Wang, M. C., Zhang, G., \& Chang, M. (2006). Analysis of superalloy turbine blade tip cracking during service. Engineering Failure Analysis, 13(8), 1429-1436.

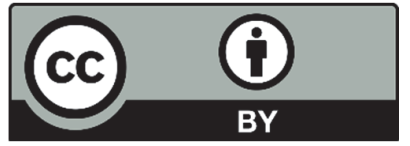

(C) 2018 by the authors; licensee Growing Science, Canada. This is an open access article distributed under the terms and conditions of the Creative Commons Attribution (CC-BY) license (http://creativecommons.org/licenses/by/4.0/). 